\title{
The Hydrolytic Destruction of Modified Cellulosic Materials in Conditions Simulating a Purulent Wound
}

\author{
Vaniushenkova Anna Alexeevna ${ }^{1}$, Ivanova Svetlana Nikolaevna ${ }^{2}$, Kalenov Sergei Vladimirovich ${ }^{1}$ (D), \\ Markvichev Nikolay Semenovich ${ }^{1}$ (D), Belov Alexey Alexeevich 1*(D)

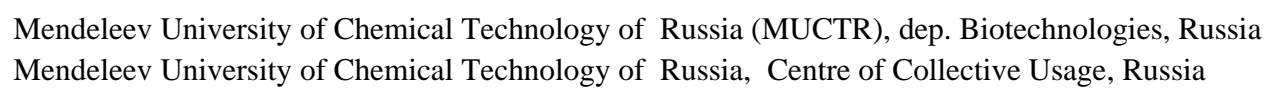

Scopus Author ID 16445532800

Received: 18.03.2020; Revised: 26.05.2020; Accepted: 29.05.2020; Published: 7.06.2020

\begin{abstract}
The development of new effective agents for purulent wounds treatment remains one of the most priority tasks of modern Biomedicine. An important aspect of ensuring the wound healing process is the selection of appropriate dressings. The right choice allows you to provide proper supportive therapy that accompanies the treatment of the root cause. Unlike cotton cellulose, dialdehyde cellulose undergoes significant hydrolytic and biological degradation in the human body under physiological conditions. Modern dressings are developed and produced on the basis of dialdehyde cellulose, chitosan, and various therapeutic agents (including enzymes). The kinetics of hydrolytic destruction of materials based on modified cellulose was studied. Using UV- and IR- spectroscopy, and dynamic light scattering methods, the decomposition of various cellulose matrices based on cotton medical gauze $\left(1 / 15 \mathrm{M}\right.$ phosphate buffer solution, $\left.\mathrm{pH} 6.2,37^{\circ} \mathrm{C}\right)$ was studied. The properties of the cellulose carrier' degradation products also were investigated. Their biological properties have been studied. It was found that the products of hydrolytic destruction of dialdehyde cellulose have antioxidant properties. The degradation of the cellulose carrier increases with the raising of cellulose modification degree, temperature, time, and $\mathrm{pH}$ of the solution. Chitosan stabilization of the oxidized cellulose carrier was found.
\end{abstract}

Keywords: chitosan; chitosan-coated dialdehyde cellulose; proteases; immobilized enzymes; therapeutic agents; wound healing; drug design; medical application.

(C) 2020 by the authors. This article is an open-access article distributed under the terms and conditions of the Creative Commons Attribution (CC BY) license (https://creativecommons.org/licenses/by/4.0/).

\section{Introduction}

The development of new effective agents for the treatment of purulent wounds remains one of the highest priorities of biomedicine. The selection of appropriate dressings is one of the fundamental aspects of the wound healing complex process.

The right choice allows you to provide proper supportive therapy that accompanies the treatment of the root cause. There is a large number of multifarious materials and dressing techniques available for wound management, the process of both acute and chronic non-healing purulent wounds. Much attention is paid to textile-based application forms [1-3].

The primary goal is to achieve a fully healed wound surface. However, in the case of severe chronic inflammatory processes, wound treatment may also be required at the stage of preparing the patient for surgical intervention, for example, for a skin grafting [1,3-4].

The ideal dressing material accelerates wound healing. It reduces the loss of proteins, electrolytes, and fluid from the wound helps to minimize pain (due to the introduction of 
anesthetic), and inactivate the infection (due to biocide), has cleansing (hydrolases and chemisorbents) and antioxidant properties. Wound care is constantly changing (there is new knowledge about the course of the wound healing process and the effect of various substances on it), and new products are being introduced to the market, and you need to be aware of their effect on wound healing $[1,3,5]$. When using immobilized drugs of various therapeutic agents (TA) in medical practice, it is necessary to know the mechanisms of their work and utilization in the body, and for this, it is necessary to know the structure and properties of the compositions used $[1,6-8]$.

In our previous studies [2, 6-9], the choice of oxidized by periodate cellulose (DAC) with chitosan immobilized on it was justified as a TA carrier. It should be noticed that wound dressings are disposable materials with a small exploitation term (about 24 hours, but not over 72 hours), so the biological activity should be completely realized by this time $[2,6]$.

Based on these considerations, we studied the destruction of a variety of cellulose matrices based on cotton medical gauze in $1 / 15 \mathrm{M}$ phosphate buffer (PB) solution ( $\mathrm{pH} 6.2$, $37^{\circ} \mathrm{C}$ ). The properties of degradation products of cellulose carriers, including their biological properties, have also been studied.

\section{Materials and Methods}

\subsection{Materials.}

In this work we used: cellulose such as medical gauze (cellulose) (RF Standart GOST 9412-93); chitosan(Ct) from crab shell (Bioprogress, Schelkovo, Moscow region, Russia, humidity $10 \%$, deacetylation degree $80.0 \%$, kinematic viscosity $383.7 \mathrm{cSt}, \mathrm{MW} \approx 500 \mathrm{kDa}$ ). Miramistin (Mir) (Moscow, Russia) was used; 3,5-dinitrosalicylic acid (DNSA) (SigmaAldrich, USA). All other chemicals were of analytical grade, and no further purification was required.

\subsection{Apparatus and instruments.}

UV-Vis spectra were measured on the recording spectrophotometer Shimadzu UV2600 (Japan).

Zeta $(\zeta)$ potential and the average particles' diameter were measured on a Zetasizer Nano ZS, Malvern Instruments, (UK).

IR spectra were obtained using a spectrometer Nicolet 380 with the FTIR (frustrated total internal reflection) (Thermo Scientific, USA). (CCU MUCTR).

Scanning Electron Microscopy (SEM). The surface of the preparations was investigated with the JSM JEOL 6510LV microscope (JEOL, Japan). Samples were prepared by depositing a layer of platinum with a thickness of $10 \mathrm{~nm}$ on a JFC-1600 magnetron sputtering unit (JEOL, Japan). The deposition was carried out in a vacuum (5 Pa) at a current of $30 \mathrm{~mA}$ and a duration of 20 seconds. Argon was used as a purge gas. The samples were taken in the mode of secondary electrons with an accelerating voltage of $15 \mathrm{kV}$. (CCU MUCTR).

\subsection{The obtaining of immobilized materials.}

We activated our cellulose materials by the periodate oxidation, as it was shown in [610]. The number of aldehyde groups on the carrier we determined using a 3,5-dinitrosalicylic acid (DNSA), a calibration curve was constructed using glucose [11]. The materials without 
chitosan we obtained following these steps: TA solution was immobilized on the cellulose carrier and was air-dried. To obtain chitosan-containing materials we immobilized chitosan gel on the cellulose, and after the air-drying process, we immobilized our TAes from the water solutions, or we just immobilized a TAes-containing chitosan gel right on the cellulose matrix [6]. Dried materials with a residual humidity no more than $6 \%$ were stored in polyethylene bags at room temperature in a dark place. In our studies, we controlled a biological activity of obtained immobilized samples.

\subsection{Determination of $A O A$.}

To determine the antiradical activity (AOA) of our preparations, we used the reaction with a stable free radical 2,2-diphenyl-1-picrylhydrazyl (DPPH) [12]. The studies were carried out using a Shimadzu UV-2600 recording spectrophotometer at a wavelength of $517 \mathrm{~nm}$ in 1 $\mathrm{cm}$ wide cuvettes. A solution of DPPHs $(0.2 \mathrm{mmol} / \mathrm{l})$ manufactured by Aldrich was prepared in $96 \%$ ethanol. To $2 \mathrm{ml}$ of each of the obtained solutions of the series, $2 \mathrm{ml}$ of the DPPH solution was poured and, after mixing after 30 minutes or another specified time, the values of optical density were recorded at $\lambda=517 \mathrm{~nm}$. As control samples, pure solvents were used in which working solutions were prepared. Working solutions were prepared by the method of sequential dilutions.

We used only freshly prepared solutions. All protein solutions in which turbidity was formed were centrifuged at $\mathrm{c} / \mathrm{f}$ at $20^{\circ} \mathrm{C}$ for 20 minutes at $14,000 \mathrm{rpm}$. The formula was calculated \%inhibition:

$\%$ Inhibition $=(A C(0)-A A(t) /(A C(0))) \cdot 100$,

$\mathrm{AC}(0)$ is the optical density of the control sample, $\mathrm{AA}(\mathrm{t})$ is the optical density of the sample. 1 Antioxidant activity unit (AOA) is the amount of the drug that causes a 50\% inhibition of the DPPH solution under special conditions.

\subsection{Determination of hydrolytic destruction.}

To study the kinetics of hydrolytic degradation of materials, the sample was poured into $1 / 15 \mathrm{M} \mathrm{PB}, \mathrm{pH} 6.2$ with a hydro module (usually $\approx 50)$, and placed in a water thermostat $\left(37^{\circ} \mathrm{C}\right.$ ) with periodic stirring; the required parameter was determined at specified intervals. The change in various physicochemical characteristics of the carriers and their degradation products was studied (there were studied both the carriers themselves and the solutions in which they were placed). As the measured parameters, UV spectra and IR spectra, hydrodynamic radii, and $\zeta$ potentials were measured, reducing substances and polysaccharides were determined using quantitative reactions.

\subsection{Statistical analysis.}

All experiments in this study were performed in triplicate, if not specifically noted. Analysis of variance (ANOVA) was performed with the Matlab program version 8.4 (USA). The results are expressed as mean \pm standard deviation (SD), and the least significant differences for comparison of means were computed at $\mathrm{p}>0,95$.

\section{Results and Discussion}

While cellulose (under physiological conditions) is insoluble in water and resistant to weak solutions of acids and alkalis, dialdehyde cellulose (DAC is a product of periodate 
oxidation of cellulose) and its derivatives undergo destruction in water and weakly acidic, and they degraded more in weakly alkaline solutions [2,13 -15].

This process is called hydrolytic destruction. The kinetics of hydrolytic destruction is described by semi-logarithmic anamorphosis, which allows us to calculate the hydrolytic destruction rate constants as the rate constant of first-order reactions. It can be calculated (and most importantly, to regulate by changing the degree of oxidation) for what time half of all DAC - TA fragments capable of transitioning into a soluble state diffuse into wound exudate. This is extremely important for the practical application of therapeutic systems in medical practice. Cellulose $(\mathrm{C})$ is a stereoregular, highly oriented crystalline polymer.

The degree of cotton' crystallinity is $65-70 \%$. During periodate oxidation, the amorphous part is primarily converted to the DAC. The crystalline part remains a frame, which, at relatively low oxidation states, provides sufficient mechanical strength for the DAC $[6,13]$. But as shown in [6.16], with an increase in the degree of the oxidation of DAC, the mechanical strength of the modified material decreases. When stored (at room temperature, in the absence of light, the residual moisture of the material is not more than 10\%), the mechanical strength of oxidized cellulose decreases further, and many those who are immobilized on DAC-TA can contribute to this.

During hydrolytic destruction of cellulose itself resulting from the exposure to hydrolyzing agents, there is a break in glycosidic bonds and a decrease in the cellulose polymerization degree $[13,17]$ according to the scheme:

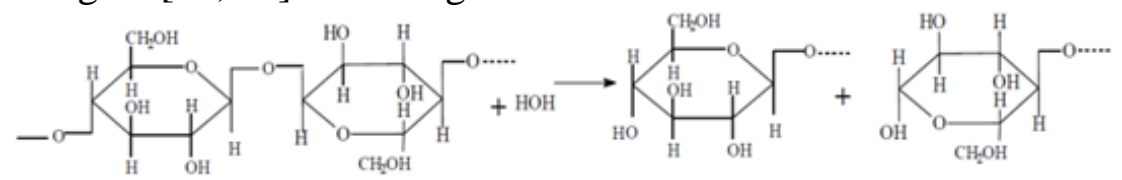

Figure 1. Possible occurrence of reducing ends in the cellulose molecule.

According to the authors of [17], there is the possibility of splitting off a small number of links (not more than 50-60) from the reducing end of a macromolecule by the reaction of the so-called pilling. The reason for such destruction is considered to be a weakening of the $\beta$ glycosidic bond during aldol condensation initiated by the aldehyde group in the presence of hydroxyl ions.

At the site of breaking of the glycosidic bond 1-4, one link forms a free glycosidic hydroxyl, i.e., the aldehyde group (at the 1st atom of C), and the other link - alcohol hydroxyl (at the 4-th atom of C). The kinetics of hydrolytic destruction can be traced to an increase in the reducing ability of the reaction mixture or to a decrease in the average degree of cellulose polymerization. The same applies to DAC derivatives containing or not various TA. The authors of [14-16] suggested that the degradation of DAC in an aqueous medium proceeds to 2,4-dihydroxybutanoic acid and glycolic acid.

The process of destruction of DAC -based carriers begins immediately when they are placed (or preparations based on them) in a solution $\left(37^{\circ} \mathrm{C}, \mathrm{pH} 6.2\right)[6,8,9,13]$. This is clearly seen in the spectra of cellulose carriers shown in Figures 2-5 (the number of aldehyde groups $\mathrm{mM} / \mathrm{g}$ is indicated in brackets).

Moreover, all carriers used by us are susceptible to hydrolytic destruction (Figure 2). Since for the DAC sample (1.20) the content of destruction products that have an intensive absorption in the studied region is too large, to see the real spectral picture, we had to dilute the initial sample by 1/15M PB 6.2 in 10 times (DAC (1.20) d 10). As can be seen from Figure 2 , unmodified cellulose also releases into water-soluble solution fragments absorbing in the 
UV region $\left(37^{\circ} \mathrm{C}, \mathrm{pH} 6.2\right)$. In the human body, the hydrolytic destruction often occurs in conjunction with biological destruction under the influence of enzymes, as well as purulentnecrotic exudate. In this case, both the carrier itself and immobilized TAs, as well as the connections (links) between them, may be affected.

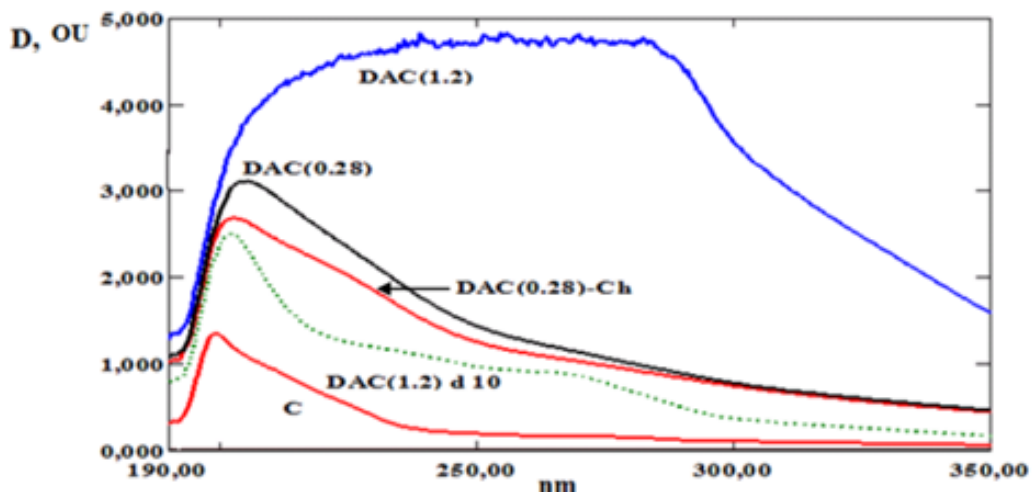

Figure 2. UV spectra of solutions of cellulose carriers in 1/15M PB 6.2 at $37^{\circ} \mathrm{C}$ after $550 \mathrm{~h}(\mathrm{~d} 10$-diluted 10 times DAC solution (1.20)).

During the hydrolytic destruction of the cellulose matrix decomposition products of different nature can be released into the aqueous medium, [13,14,15]. Moreover, several authors $[13,16]$ suggest that the cellulose regions where the oxidized polysaccharide units are located will first be subjected to destruction. According to the authors of [14-19], it is very difficult to strictly identify the products of hydrolytic decomposition of cellulose matrices.

The composition of the degradation products is affected by the source of cellulose, the conditions for obtaining the fiber (material), temperature, the composition of the medium, the presence of various substances, etc.

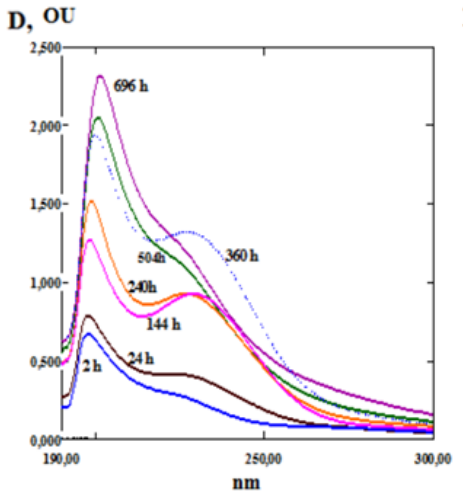

(a)

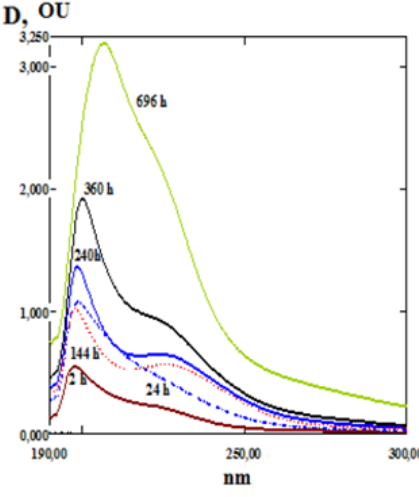

(b)

Figure 3. UV spectra of modified cellulosic materials after aging in $1 / 15 \mathrm{M} \mathrm{PB}(\mathrm{pH} 6.2)$ at $37^{\circ} \mathrm{C}$ for various times (pH 6.2): a) DAC (0.3) samples; b) DAC (0.3)-Ct samples.

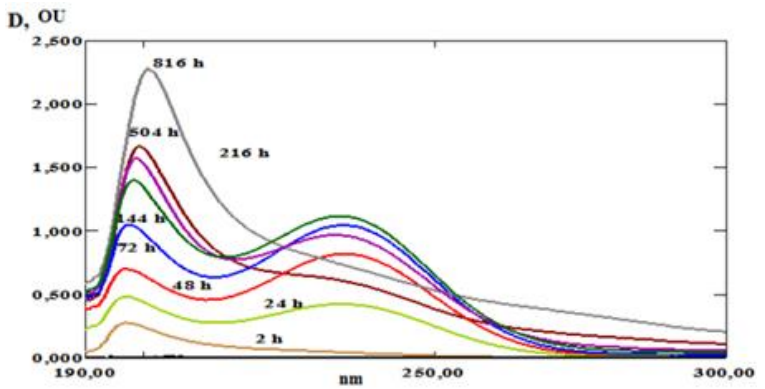

Figure 4. UV spectra of solutions (diluted 10 times) DAC (1.20) in $1 / 15 \mathrm{M} \mathrm{PB} 6.2$ at $37^{\circ} \mathrm{C}$ for various times. 


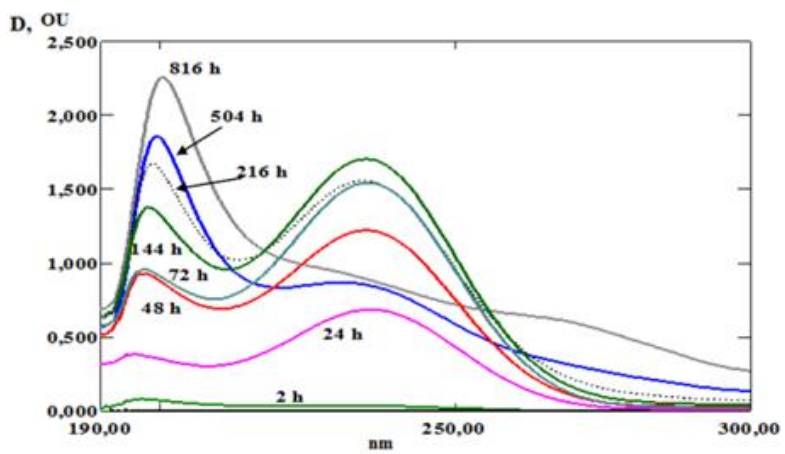

Figure 5. UV spectra of solutions (diluted 10 times) DAC (1.20)-Ch in $1 / 15 \mathrm{M}$ PB 6.2 at $37^{\circ} \mathrm{C}$ for various times.

As can be seen from the presented data, the peak in the region of $240 \mathrm{~nm}$ associated with the presence of aldehyde groups $[14,15]$ initially increases and then decreases; this is especially noticeable over time for a greater degree of oxidation $(1.2 \mathrm{mmol} / \mathrm{g})$. This assumption is confirmed by studies $[14,16]$ and the data of Figures $7-10$. It could occur to the destruction of the oxidized material (the bonds will break near or along with the oxidized link) firstly.

The aldehyde-containing fragments of the carrier will enter into the solution, and then they will probably transform (one of the possible ways is shown in [15]). These assumptions are confirmed by received data about average hydrodynamic diameters (d) and the Zeta potential $(\zeta)$ cellulose and its derivatives (Table 1 and Figure 5).

Table 1. The average hydrodynamic diameters $(d)$ and the zeta potential $(\zeta)$ of cellulose and its derivatives, $d$,

\begin{tabular}{|c|c|c|c|c|}
\hline \multirow[b]{2}{*}{ Sample } & \multicolumn{2}{|c|}{ d. $\mathrm{nm}$} & \multicolumn{2}{|c|}{$\zeta . \mathbf{m V}$} \\
\hline & $4 \mathrm{~h}$ & $24 \mathrm{~h}$ & $4 \mathrm{~h}$ & $24 \mathrm{~h}$ \\
\hline Cellulose (0.008) & $\begin{array}{c}669.53(97.13 \%) \\
5447(2.7 \%)\end{array}$ & $\begin{array}{l}1034(80 \%) \\
5325(20 \%)\end{array}$ & -2.58 & -7.47 \\
\hline DAC $(0.3)$ & $\begin{array}{c}450.43(54.57 \%) \\
4574(45.43 \%)\end{array}$ & $600.07(100 \%)$ & -14.90 & -9.27 \\
\hline DAC (1.0) & $\begin{array}{c}667.33(63.23 \%) \\
4636(23.85 \%) \\
3.90(11.93 \%) \\
46.40(1 \%) \\
\end{array}$ & $\begin{array}{c}385.07(47.7 \%) \\
3.305(30.5 \%) \\
4526(21.77 \%)\end{array}$ & -18.00 & -11.00 \\
\hline $\mathrm{DAC}(0.3)-\mathrm{Ct}$ & $\begin{array}{c}3868(54 \%) \\
659.73(42.7 \%) \\
0.709(2.3 \%) \\
144.7(1 \%)\end{array}$ & $962.37(100 \%)$ & -14.00 & -9.24 \\
\hline
\end{tabular}

* for the cellulose derivatives in brackets, the number of aldehyde groups, $\mathrm{mM} / \mathrm{g}$

The primary cause of the appearance of a negative charge on the surface of cellulose fibrils placed in an aqueous medium is supposed to be the dissociation of all electrolytically active groups on the fibers' surface, as well as the ionization of carboxyl groups into which aldehyde and hydroxyl groups are able to oxidize [20]. Also, A.S. Smolin et al. [20] put forward a theory of oriented solvent adsorption, which indicates to the creation of a strong negative charge on the cellulose surface due to the orientation of water dipoles.

Thus, a decrease in the Zeta potential for all the studied samples (Table 1) confirms the conclusion about the breakdown of cellulose carriers under the studied conditions. Some studies $[13,17,21]$ talk about the isolation from the matrix of unoxidized cellulose (medical gauze) compounds formed during the preparation and processing of tissue fiber, which we explain by the decreasing in the value of the solution potential where the unmodified cellulose sample was placed. The initial value of the Zeta potential is expected to be lower for DAC with a higher oxidation degree. It should also be taken into consideration that the potential of DAC- 
chitosan practically does not differ from the value for DAC (0.28), which indicates to the nature of the binding of the chitosan amino groups to the aldehyde group of the oxidized matrix, which was described in detail in our work [10]. Based on the data obtained, we can state that large conglomerates of polysaccharides formed at the first moment are subjected to hydrolytic destruction over time.

The absence of particularly small particles can be explained by the screening effect of large aggregates or fragments of the matrix transferred to the solution as a result of hydrolytic destruction. This process becomes especially intensive with an increasing degree of carrier oxidation, exposure time, $\mathrm{pH}$ of the medium, and temperature [13].

Figure 6 and [8] show data on the hydrolytic degradation of cellulosic materials by using 3,5-dinitrosalicylic acid (DNSA) [11]. Moreover, both the number of aldehyde groups on the carrier, and the number of reducing substances formed in the solution, or the total amount of reducing substances (on the carrier and transferred to the solution) were determined during various times of keeping cellulose materials in the $1 / 15 \mathrm{MPB}(\mathrm{pH} 6.2)$ at $37^{\circ} \mathrm{C}$. The degradation products of $\mathrm{C}$ and DAC were also studied by the phenol-sulfur method [28].

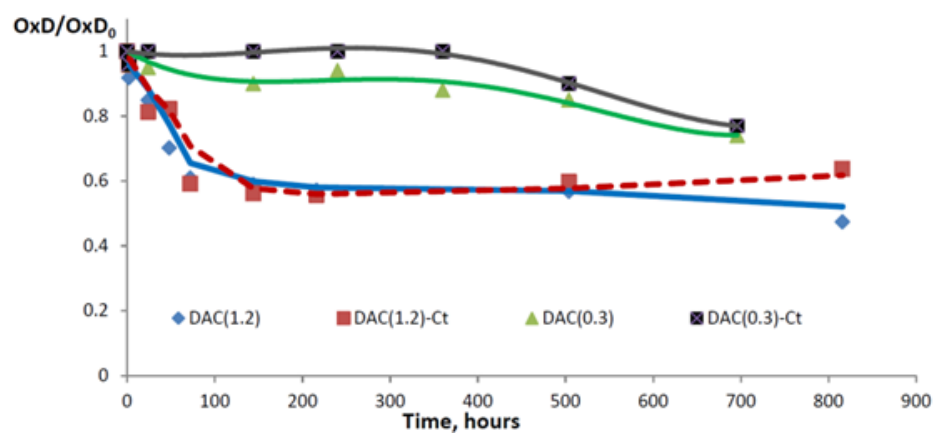

Figure 6. The change in the content of aldehyde groups on the carrier for different exposure times in $1 / 15 \mathrm{M} P B$ (pH 6.2) at $37^{\circ} \mathrm{C}$; for 1 , the value of $\mathrm{OxD}_{0}$ is taken before the samples are placed in the solution.

A decrease in the number of reducing groups for oxidized cellulose over time is observed (Figure 6), which indicates a gradual decomposition of the matrix as a result of hydrolytic destruction. And with a higher content of aldehyde groups, the process proceeds more significantly. Moreover, in solutions in which oxidized cellulose carriers were placed, an increase in reducing substances is observed over time [8.28]. The total decrease in reducing groups [8] can result from the phenomenon that low-molecular-weight fragments of the matrix, the aldehyde groups of which exist in the aqueous solution in the form of hemiacetals, are disproportionate to carboxyl and hydroxyl groups, and over time are accumulated in the solution in the form of low-molecular-weight carboxylic acids, which were determined by HPLC method.

The authors of $[14,15,29]$, described the formation of carboxylic acids during biodegradation. The same conclusions were reached by the authors of $[14,15,29]$, who described the formation of carboxylic acids during biodegradation of periodate acid-oxidized cellulose. Immobilization of $\mathrm{Ct}$ on a cellulose carrier stabilizes DAC. As we have established, the samples of $\mathrm{Ct}$ used by us (regardless of the presence of 1/15M PB in the solution) do not interact with DNSA (there are no reducing groups). For immobilized preparations of DACchitosan, there is a slight increase in the number of reducing substances without subsequent changes throughout the experiment. This fact is associated with the interaction of the aldehyde groups of the oxidized cellulose carrier with the amino groups of chitosan, which prevents the described matrix decomposition and (or) the appearance of carbonyl groups, respectively, 
reducing the number of reducing groups. As it was shown in [30], when the Ct solution interacts with low molecular weight aldehydes (formaldehyde, acetic aldehyde, glutaraldehyde), all Ct amino groups are bound to the aldehyde, but when $\mathrm{Ct}$ interacts with high molecular weight dialdehydes (DAC, dialdehyde starch) binds no more than $20 \%$ of free amino groups.

The low-molecular-weight aldehydes released during the destruction (fragments of the deep degradation of $\mathrm{C}$ carriers) can be linked by $\mathrm{Ct}$ amino groups. The mechanical strength of DAC $[6,13,23]$ decreases with increasing modification' degree, time, and storage conditions.

Despite the fact that a very significant number of works $[13,15,16,22,31]$ are devoted to IR spectroscopy of cellulose and its derivatives, the obtained results using this method remain rather modest. This is due to the complexity of the interpretation of the IR spectra of monosaccharides and the numerous experimental difficulties caused by the characteristics of monosaccharides, not to mention polysaccharides.

Absorption bands characteristic of a particular monosaccharide and unique to it do not exist. Therefore, for the identification, by comparing with the spectrum of a known compound, it is necessary to take the IR spectrum in a wide frequency range, usually from 4000 to 650 $\mathrm{cm}^{-1}$, and the region of $1250-650 \mathrm{~cm}^{-1}$, the so-called fingerprint region, is especially characteristic.

As an example figures, 7-10 show the IR spectra of samples C, DAC, and DAC-Ct before and after exposure to $1 / 15 \mathrm{M} \mathrm{PB}(\mathrm{pH} 6.2)$ at $37^{\circ} \mathrm{C}$. For samples after holding (ah) in $1 / 15 \mathrm{M}$ PB solution may contain products of cellulose destruction products. Summing up the data obtained, primarily for DAC samples, significant changes in the spectra are visible in the $1800-1600 \mathrm{~cm}^{-1}$ region.

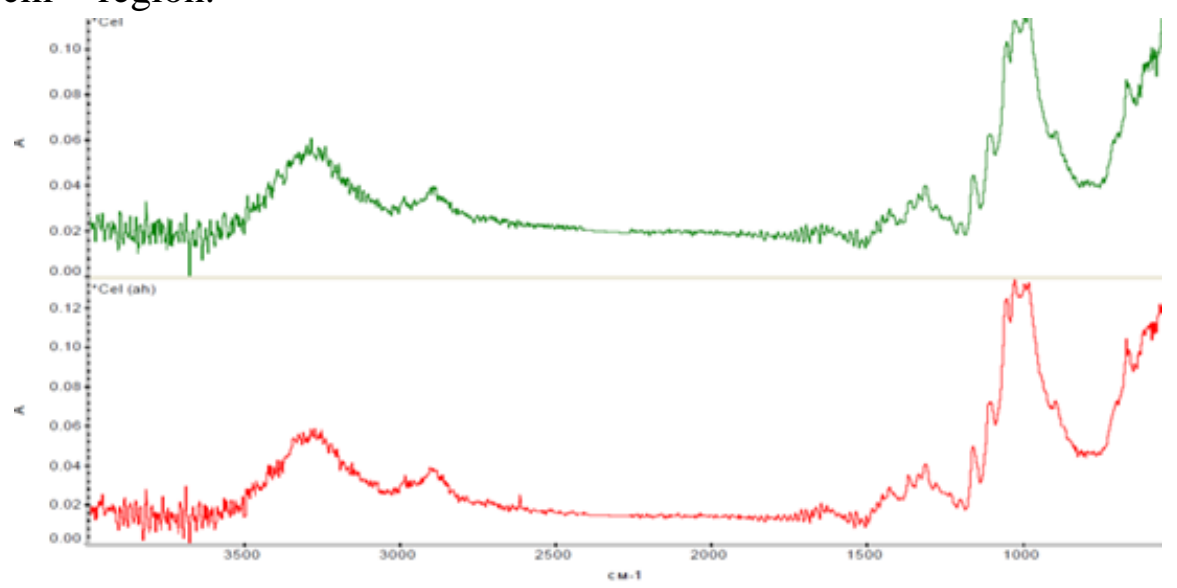

Figure 7. FTIR spectra of cellulose samples before and after aging ( $\mathrm{Cel}$ ah) in 1/15M PB (pH 6.2) at $37^{\circ} \mathrm{C} 48 \mathrm{~h}$.

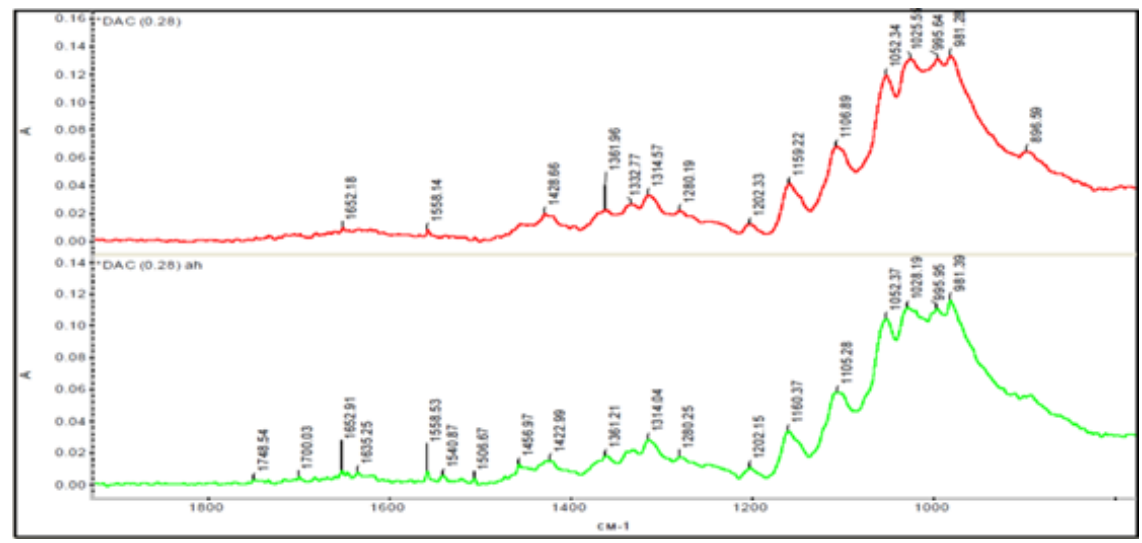

Figure 8. FTIR spectra of DAC (0.28) samples before and after aging (DAC (0.28) ah) in 1/15 M PB (pH 6.2) at $37^{\circ} \mathrm{C} 48 \mathrm{~h}$. 


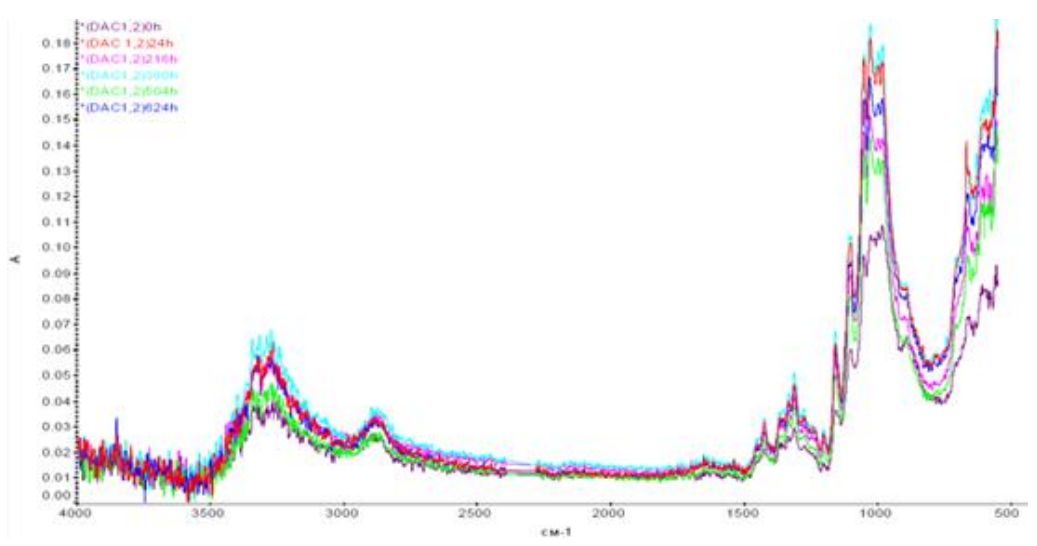

Figure 9. FTIR spectra of DAC (1.2) samples before and after aging (DAC (1.2) ah) in 1/15 M PB (pH 6.2) at $37^{\circ} \mathrm{C} 48 \mathrm{~h}$

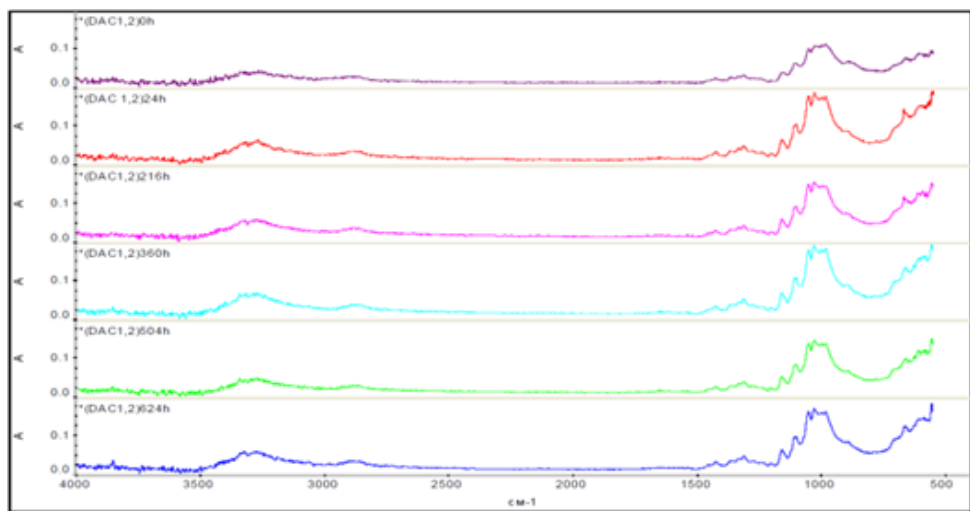

Figure 10. FTIR spectra of samples before DAC (1.2) and after holding (DAC (1.2) ah) in 1 / 15M PB (pH 6.2) at $37^{\circ} \mathrm{C}$

From the literature data $[13,16,23]$, it is known that the characteristic bands of DAC appeared in the 1740 and $880 \mathrm{~cm}^{-1}$ regions. They increased from a small shoulder to a distinct peak with increasing oxidation level. A diffuse band at $880 \mathrm{~cm}^{-1}$ can be assigned to the hemiacetal and hydrated form. The sharp peak at $1740 \mathrm{~cm}^{-1}$ is a characteristic band of carbonyl groups. It was shown [22-25] that this spectral region corresponds to aldehyde groups (including their derivatives) formed as a result of periodic oxidation of cellulose. The crystallinity index (CI) acquired by FTIR, was evaluated from the ratios of the absorption bands at $1429 \mathrm{~cm}^{-1}$ and $893 \mathrm{~cm}^{-1}$ (A1429/A893) [24,26].

The data obtained show the decomposition of the aldehyde-containing sections of cellulose, the transition into the solution of various active fragments of the oxidized matrix that undergo a further transformation.

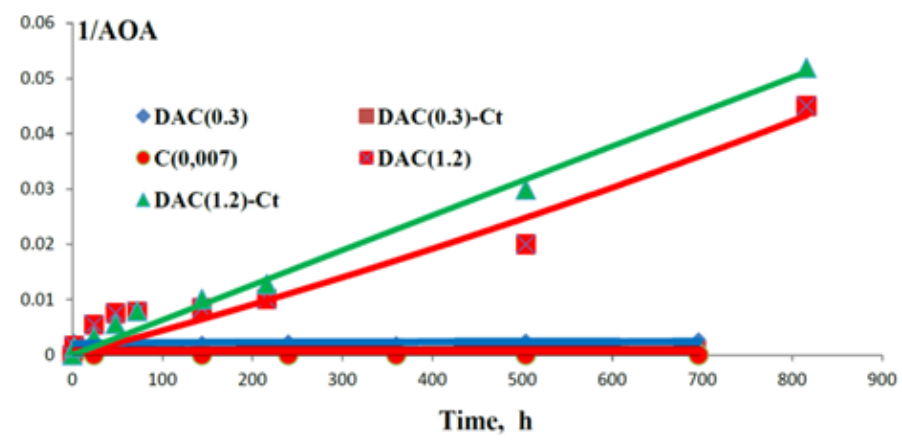

Figure 11. The change in the antioxidant activity of the solution during various exposure times in $1 / 15 \mathrm{M} \mathrm{PB}(\mathrm{pH} 6.2)$ at $37^{\circ} \mathrm{C}$.

As we established earlier, the products of hydrolytic degradation of the DAC have interesting and useful properties from the perspective of wound healing. They have antioxidant 
properties and slow down the growth of pathogenic microorganisms such as St. aureus and E.coli $[23,24]$, i.e., we can say that DAC is a prodrug. The data obtained are presented in Figures 11 and 12. It should be borne in mind that the carriers themselves, regardless of the oxidation degree (the number of aldehyde groups), as well as cellulose and its solutions do not possess AOA [32].

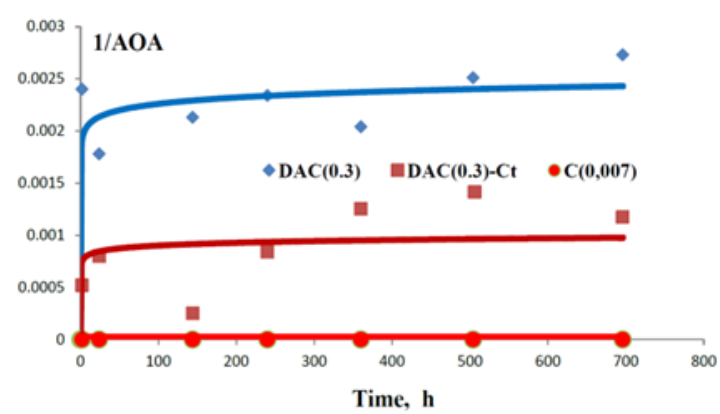

Figure 12. The change in the antioxidant activity of solution $\mathrm{C}, \mathrm{DAC}, \mathrm{DAC}-\mathrm{Ct}$ during different exposure times in $1 / 15 \mathrm{M} \mathrm{PB}(\mathrm{pH} 6.2)$ at $37^{\circ} \mathrm{C}$

The aging of $\mathrm{C}$ and DAC in ethyl alcohol or DMSO showed an almost complete absence of AOA regardless of the aging time. The presence of AOA in DAC samples are caused only by hydrolytic destruction of oxidized cellulose. Moreover, the type of AOA changes dependence is almost identical to the number of occurrences of reducing groups in solution. Immobilized $\mathrm{Ct}$ prevents the destruction of oxidized cellulose and reduces the value of AOA degradation products. The analysis of the obtained dependencies showed that the AOA DAC grows during the entire study. These results can be explained with the involvement of previously obtained data given in [27, 28].

According to [15], as well as the scheme of DAC degradation under the conditions described by us (Figure 1), the hydrolytic degradation of cellulose proceeds through the formation of intermediate products, which overtime pass into more stable forms, representing [18] organic compounds. This indicates the duration of the accumulation of intermediate products due to a large number of possible degradation products compared to other samples due to a significantly larger number of aldehyde groups per carrier mass. The literature provides data on the formation of a characteristic peak at $240 \mathrm{~nm}$ belonging to the enol forms of dicarboxylic compounds [33], while absorption at 275-285 nm, corresponding to free aldehyde groups, is absent (Figures 2-5).

Thus, in our opinion, the increase in the total antioxidant activity of the solutions in which the cellulose carriers were located is associated with the accumulation of intermediate products in the medium, which, as already noted, are converted into various organic compounds.

Our additional experiments confirm the presence of antioxidant activity in some carboxylic acids, which allows us to conclude that the growth of antioxidant activity is precisely due to an increase in the concentration of carboxylic acids in the aqueous medium, while insufficient to change the $\mathrm{pH}$ of the buffer solution.

The determining role in wound healing belongs to the carrier on which the drug is immobilized. The speed and completeness of the release of the active substance in the body, the degree of the therapeutic effectiveness of the drug [1,3,34,35], depends on it. One of the most important properties of wound healing materials is biodegradability and biocompatibility. The modified (oxidized) cellulose carrier undergoes biodegradation under the influence of 
environmental conditions, the human body (enzymes, $\mathrm{pH}$ ). These reactions proceed without the formation of any toxic products [6,7].

The molecular structure of cellulose allows you to create material based on it, capable of various sorbing compounds, such as toxins, microorganisms, and wound exudate. The great interest in cellulose as a carrier for therapeutic agents with biological activity is also explained by the phenomenon of cellulose being a highly available polymer in medicine with a relatively low cost, that is very important for the production of compositions based on it [7].

\section{Conclusions}

It was shown that when placing dialdehyde cellulose and its derivatives in a model liquid medium, the process of hydrolytic destruction of the polysaccharide begins. We believe that DAC is a prodrug. Products of hydrolytic degradation of DAC have antioxidant activity.

The DAC-based materials are not only good chemisorption carriers for creating of the wound healing preparations with immobilized TAs, but they also exhibit the properties of a biocide and an inhibitor of free radical reactions, which makes the further development of wound coatings based on them a promising direction in creating therapeutic applications for treating purulent and burn wounds.

\section{Funding}

This research received no external funding.

\section{Acknowledgments}

This research has no acknowledgment.

\section{Conflicts of Interest}

The authors declare no conflict of interest.

\section{References}

1. Ather, S.; Harding, K.G. Wound management and dressings. In Advanced textiles for wound care, 1st ed.; Rajendran, S., Ed.; Woodhead Publishing: Cambridge, UK, 2009; pp.3-19. https://doi.org/10.1533/9781845696306.1.3

2. Belov, A.A.; Vaniushenkova, A.V.; Dosadina, E.E.; Khanafina, A.A. New textile dressings based on biodegradable polymers containing proteinases for wounds and burns treatment. Wounds and wound infections. The prof. B.M. Kostyuchenok journal 2018, 5, 16-26. https://doi.org/10.25199/2408-9613-20185-1-16-26

3. Mukhopadhyay, A.; Sikka, M.P.; Midha, V.K. Speciality dressings for managing difficult-to-heal wounds. In Advanced Textiles for Wound Care, 2rd ed.; Rajendran, S., Ed.; Woodhead Publishing: Cambridge, UK, 2019; pp. 391-42. https://doi.org/10.1016/B978-0-08-102192-7.00014-X.

4. Sarabahi, S. Recent advances in topical wound care. Indian J. Plast. Surg. 2012, 45, 379-387, https://doi.org/10.4103/0970-0358.101321.

5. Frykberg, R.G.; Banks, J. Challenges in the treatment of chronic wounds. Adv. Wound Care 2015, 4, 560582. https://doi.org/10.1089/wound.2015.0635.

6. Belov, A.A. Development of industrial technologies for obtaining new medical materials based on modified fiber-forming polymers containing biologically active protein substances. Diss. on com. ac. deg. Dr. tech. Sciences M., MUCTR 2009, 385. 
7. Dosadina, E.E.; Savelyeva, E.E.; Belov, A.A. The effect of immobilization, drying and storage on the activity of proteinases immobilized on modified cellulose and chitosan. Process Biochemistry 2018, 64, $213-220$. https://doi.org/10.1016/j.procbio.2017.10.002.

8. Vaniushenkova, A.V.; Dosadina, E.E.; Belov, A.A. Synthesis and study of the properties of composite materials based on cellulose and chitosan containing various therapeutic agents. Part 3. Hydrolytic destruction of dressings based on dialdehydecellulose. Butlerov Communications 2019, 59, 47-59.

9. Belov, A.A.; Dosadina, E.E. Interaction between chitosan solutions, cellulose carriers and some of the multienzyme complexes. International Journal of Bioorganic Chemistry 2017, 2, 51-60. https://doi.org/10.11648/j.ijbc.20170202.12

10. Dosadina E.E.; Kulmetyeva M.A.; Belov A.A. The changing of enzymatic activity of hydrolases immobilized on natural polysaccharide matrix for purulent and burn wounds treatment during storing and exploitation. Biointerface research in applied chemistry 2016, 6, 1291-1298.

11. Miller, G.L. Use of Dinitrosalicylic Acid Reagent for Determination of Reducing Sugar. Anal Chem 1959, 31, 426-428. https://doi.org/10.1021/ac60147a030.

12. Glavind, J.; Jorgensen, O.; Rost, E. Antioxidants in Animal Tissue. Acta Chemica Scandinavica 1963, 17, 1635-1640. https://doi.org/10.3891/acta.chem.scand.17-1635.

13. Rogovin, Z.A. Chemistry of cellulose. Chimia: Moscow, Russia, 1972. pp. 509.

14. Singh, M.; Ray, A.R.; Verma, P.V.K.; Guha, S.K. Potential Biosoluble Carriers: Biocompatibility and Biodegradability of Oxidized Cellulose. Biomaterials, Medical Devices, and Artificial Organs 1979, 7, 495512. https://doi.org/10.3109/10731197909118964.

15. Singh, M.; Ray, A.R.; Vasudevan, P. Biodegradation studies on periodate oxidized cellulose. Biomaterials 1982, 3, 16-20. https://doi.org/10.1016/0142-9612(82)90055-2.

16. Kim, U.J.; Kuga, S.; Wada, M.; Okano, T.; Kondo, T. Periodate oxidation of crystalline cellulose. Biomacromolecules 2000, 1, 488-492. https://doi.org/10.1021/bm0000337.

17. Gubina, S.M.; Stokozenko, V.G. Study of nukleophilic ions action $\mathrm{OH}-$ and $\mathrm{HO}_{2}$ - on desruction of cotton cellulose. Russian journal of chemistry and chemical technology 2007, 50, 96-99.

18. Knill, C.J.; Kennedy, J.F. Degradation of cellulose under alkaline conditions. Carbohydrate Polymers 2003, 51, 281-300. https://doi.org/10.1016/S0144-8617(02)00183-2.

19. O'Meara, D.; Richards, G.N. Alkaline Degradation of Polysaccharides. Part V. Periodate oxycellulose. $J$. Chem. Soc. 1958, 4504-4508. https://doi.org/10.1039/JR9580004504.

20. Smolin, A.S.; Shabiev, R.O.; Jakkola, P. The research of zeta potential and cationic demand of chemical and mechanical pulps. Chemistry of vegetable raw materials 2009, 177-184.

21. Potthast A.; Rosenau T.; Kosma P. Analysis of Oxidized Functionalities in Cellulose. In Polysaccharides II. Klemm, D., Ed.; Springer: Leipzig, Germany, 2006; Volume 205; pp. 1-49. https://doi.org/10.1007/11776895.

22. Fan, Q.G.; Lewis, D.M.; Tapley, K.N. Characterization of Cellulose Aldehyde Using Fourier Transform Infrared Spectroscopy. Journal of Applied Polymer Science 2001, 82, 1195-1202. https://doi.org/10.1002/app.1953.

23. Bikales, N.M.; Segal, L. Cellulose and cellulose derivatives. 2nd ed.; Wiley-Interscience: New York, NY, USA, 1971; pp. 893.

24. Oh, S.Y.; Yoo, D.I.; Shin, Y.; Kim, H.C.; Chung, Y.S.; Park, W.H.; Youk, J.H. Crystalline structure analysis of cellulose treated with sodium hydroxide and carbon dioxide by means of X-ray diffraction and FTIR spectroscopy. Carbohydrate Research 2005, 340, 2376-2391. https://doi.org/10.1016/j.carres.2005.08.007.

25. Liu, X.; Wang, L.; Song, X.; Zhao, J.R.; Wang, S. A kinetic model for oxidative degradation of bagasse pulp fiber by sodium periodate. Carbohydrate Polymers 2012, 90, 218-223. https://doi.org/10.1016/j.carbpol.2012.05.027.

26. Hou, Q.X.; Liu, W.; Liu, Z.H.; Bai, L.L. Characteristics of wood cellulose fibers treated with periodate and bisulfate. Industrial \& Engineering Chemistry Research 2007, 46, 7830-7837. https://doi.org/10.1021/ie0704750.

27. Vaniushenkova, A.A.; Dosadina, E.E.; Hanafina, A.A.; Ivanova, S.N.; Kalenov, S.V.; Markvichev, N.S.; Belov, A.A. Synthesis and study of the properties of composite materials based on cellulose and chitosan containing various therapeutic agents. Part 1. Based on cellulose and various therapeutic therapeutic agents. Composites of chitosan composites. Butlerov Communications 2019, 57, 130-143.

28. Vaniushenkova, A.A.; Dosadina, E.E.; S.N.; Kalenov, S.V.; Belov, A.A. Synthesis and study of the properties of composite materials based on cellulose and chitosan containing various therapeutic agents. Part 2. Effect 
of chitosan on the destruction of cellulosic carriers and the kinetics of release of the therapeutic agent in the model environment. Butlerov Communications 2019, 57, 105-119.

29. Devi, K.S.; Sinha, T.J.M.; Vasudeva, P. Biosoluble surgical material from 2,3-diadehyde cellulose. Biomaterials 1986, 7, 193-196. https://doi.org/10.1016/0142-9612(86)90101-8.

30. Chernyshova, E.B. Modification of film materials based on chitosan low molecular weight and polymeric aldehydes. Diss. on the competition uch. step. Cand. chemical Sciences Volgograd 2018, 113.

31. Zhbankov, R.G.; Marunov, R.M.; Ivanova, N.V.; Shishko, A.M. Spectroscopy of cotton. Science: Moscow, Russia, 1976; pp. 248.

32. Chang, S.H.; Wu, C.H.; Tsai, G.J. Effects of chitosan molecular weight on its antioxidant and antimutagenic properties. Carbohydrate Polymers 2018, 7181, 1026-1032. https://doi.org/10.1016/j.carbpol.2017.11.047.

33. Drobchenko, S.N.; Isaev-Ivanova, L.S.; Kleiner, A.R. Aldo-enol tautomerism of dialdehydedextrans. Journal of High Molecular Compounds 1991, 33, pp.220-224.

34. Pandey, S.P.; Shukla, T.; Dhote, V.K.; Mishra, D.K.; Maheshwari, R.; Tekade, R.K. Chapter 4-Use of Polymers in Controlled Release of Active Agents. In Basic Fundamentals of Drug Delivery, 1st ed.; Rakesh, T., Ed.; Academic Press: London, UK, 2019; pp. 113-172. https://doi.org/10.1016/B978-0-12-8179093.00004-2.

35. Huang et al. Polymeric Carriers for Controlled Drug Delivery in Obesity Treatment. Trends in Endocrinology \& Metabolism 2019, 30, 974-989. https://doi.org/10.1016/j.tem.2019.09.004. 